\title{
ON THE SPECTRUM \\ OF THE NEUMANN LAPLACIAN OF LONG RANGE HORNS: A NOTE ON THE DAVIES-SIMON THEOREM
}

\author{
V. JAKŠIĆ
}

(Communicated by Theodore Gamelin)

\begin{abstract}
For a class of regions with cusps (e.g., $\Omega=\{(x, y): x>1$, $\left.\left.|y|<\exp \left(-x^{\alpha}\right)\right\}, 0<\alpha<1\right)$ we show that $\sigma_{\mathrm{ac}}\left(-\Delta_{N}^{\Omega}\right)=[0, \infty)$ of uniform multiplicity one, $\sigma_{\text {sing }}\left(-\Delta_{N}^{\Omega}\right)=\varnothing$, and $\sigma_{\mathrm{pp}}\left(-\Delta_{N}^{\Omega}\right)$ consists of a discrete set of embedded eigenvalues of finite multiplicity.
\end{abstract}

\section{INTRODUCTION}

This note is a contribution to the study of spectral properties of Neumann Laplacians on regions of the form

$$
\Omega=\{(x, y): x>1,|y|<f(x)\},
$$

where $f$ is a strictly positive $C^{2}[1, \infty)$ function. The Neumann Laplacian on $\Omega,-\Delta_{N}^{\Omega}=H$, is a unique selfadjoint operator in $L^{2}(\Omega)$ whose quadratic form is given by the closure of

$$
q(\phi, \phi)=\int_{\Omega}|\nabla \phi|^{2} d x
$$

on $C_{0}^{2}(\bar{\Omega})$. The spectral properties of $H$ in regions (1.1) have been studied in $[2,5,8]$ and, if specialized to the case when $f(x)=\exp \left(-x^{\alpha}\right), \alpha>0$, they can be stated as follows $\left(f \sim g\right.$ stands for $\left.\lim _{x \rightarrow \infty} f(x) / g(x)=1\right)$.

Theorem 1.1. (i) [5] $H$ has a discrete spectrum iff $\alpha>1$.

(ii) [8] If $N_{E}(H)$ denotes the number of eigenvalues of $H$ which are less than $E$, then for $\alpha>1$

$$
N_{E}(H) \sim E^{1 / 2+1 /(2(\alpha-1))} C(\alpha)+E \int_{1}^{\infty} \exp \left(-x^{\alpha}\right) d x
$$

where

$$
C(\alpha)=\frac{1}{4(\alpha-1) \sqrt{\pi}}\left(\frac{\alpha}{2}\right)^{1 /(1-\alpha)} \frac{\Gamma(1 /(2(\alpha-1)))}{\Gamma(3 / 2+1 /(2(\alpha-1)))} .
$$

Received by the editors August 26, 1991 and, in revised form, March 1, 1992.

1991 Mathematics Subject Classification. Primary 58G25.

Research supported by Sloan Doctoral Dissertation Fellowship. 
(iii) [2] If $\alpha=1, \sigma_{\mathrm{ac}}(H)=\left[\frac{1}{4}, \infty\right)$ of uniform multiplicity one, $\sigma_{\mathrm{sing}}(H)=\varnothing$, and $\sigma_{\mathrm{pp}}(H)$ consists of a discrete set $0=\lambda_{0}<\lambda_{1} \leq \cdots \leq \lambda_{n} \rightarrow \infty$ of eigenvalues of finite multiplicity.

(iv) [2] If $0<\alpha<\frac{1}{2}$, the results of part (iii) remain valid except that now $\sigma_{\mathrm{ac}}(H)=[0, \infty)$.

In this paper we prove a theorem which, specialized to the above example, covers the range $\frac{1}{2} \leq \alpha<1$; namely, we will show that all the conclusions of Theorem 1.1(iv) are valid for $0<\alpha<1$. Thus, at least in the above special case, we have a complete picture of what happens with the Neumann Laplacian (for some recent examples of surprising spectral properties of $-\Delta_{N}^{\Omega}$, even for bounded regions $\Omega$, we refer to $[6,14])$.

Our strategy (and our proof) follows closely the one of Davies and Simon [2]. The major observation made in [2] is that any spectral behavior which distinguishes $H$ from the Dirichlet case (e.g., existence of a continuous spectrum) can come only from the subspace $P$ consisting of functions which depend on the $x$ variable only and which are in the form domain of $H$ (such functions cannot be in the form domain of Dirichlet Laplacian). Restricted to such a subspace, the form (1.1) acts as

$$
2 \int_{1}^{\infty}\left|u^{\prime}(x)\right|^{2} f(x) d x
$$

and viewed as a form on $L^{2}([1, \infty), 2 f(x) d x)$ yields an operator which is unitarily equivalent to the one-dimensional Schrodinger operator on $L^{2}([1, \infty), d x)$ of the form

$$
H_{V}=-\frac{d^{2}}{d x^{2}}+V(x), \quad V=\frac{1}{4}\left(\frac{f^{\prime}}{f}\right)+\frac{1}{2}\left(\frac{f^{\prime}}{f}\right)^{\prime} .
$$

On the subspace orthogonal to $P, H$ acts in effect as a Dirichlet Laplacian, and off-diagonal perturbation terms are controlled by the function

$$
k(x)=\left|f^{\prime}(x)\right|+f^{\prime}(x)^{2} / f(x) .
$$

We say that a function $g$ on $[1, \infty)$ is short-range if $g(x)=O\left(x^{-1-\varepsilon}\right)$ for some $\varepsilon>0$. Davies and Simon proved the following in [2]:

Theorem 1.2. (i) If $f \rightarrow 0$ and $k \rightarrow 0$ as $x \rightarrow \infty, H$ has discrete spectrum if and only if $\mathrm{H}_{V}$ does.

(ii) If $k$ and $V$ are short range, we have $\sigma_{\mathrm{ac}}(H)=[0, \infty)$ of uniform multiplicity, $\sigma_{\text {sing }}(H)=\varnothing$, and $\sigma_{\mathrm{pp}}(H)$ consists of a discrete set $0=\lambda_{0}<\lambda_{1} \leq \cdots \leq$ $\lambda_{n} \rightarrow \infty$ of embedded eigenvalues of finite multiplicity.

When $f(x)=\exp \left(-x^{\alpha}\right)$,

$$
V(x)=\frac{\alpha^{2}}{4} x^{2(\alpha-1)}-\frac{\alpha(\alpha-1)}{2} x^{(\alpha-2)}
$$

and Theorem 1.2 certainly yields parts (i), (iv) of Theorem 1.1. Case $\alpha=1$ is somewhat special since then $V=\frac{1}{4}$ : The argument of [2] still carries through, with the obvious shift of the essential spectrum. With some additional technical assumptions it was shown in [8] that in case (i) of Theorem 1.1 one has

$$
N_{E}(H) \sim N\left(H_{V}\right)+\frac{E}{2} \operatorname{Vol}(\Omega) .
$$


Using the semiclassical formula and (1.5) we get part (ii) of Theorem 1.1.

It is now easy to understand why $\alpha=\frac{1}{2}$ is the critical value: while for $0<\alpha<\frac{1}{2}$ the potential $V$ is short-range, for $\frac{1}{2} \leq \alpha<1$ it becomes longrange (thus the name, long-range horns), and to treat perturbations one has to use the long-range scattering theory, which tends to be technically involved (particularly in the case when $V(x) \sim x^{-\beta}, 0<\beta<\frac{1}{2}$, which corresponds to the range $\left.\frac{3}{4} \leq \alpha<1\right)$. The authors of [2] used the short-range Enss theory to treat perturbations and conjectured "that it is likely that one can modify ... [their] ... argument" to prove the analog of Theorem 1.2(ii) in the longrange case. By replacing the short-range Enss theory with the long-range one we extend Theorem 1.2 to the case where $V$ is long-range and dilation analytic. The dilation analyticity assumption, although restrictive, covers the example of Theorem 1.1 and enables a particularly simple technical treatment, thanks to the work of Perry $[10,11]$.

In the sequel, $H_{0}$ stands for the one-dimensional free Laplacian. Let $u(\theta)$ : $L^{2}(\mathbf{R}) \rightarrow L^{2}(\mathbf{R})$ be a unitary mapping defined as

$$
u(\theta) \phi(x)=\exp (\theta / 2) \phi(\exp (\theta) x)
$$

A potential $\widehat{V}$, defined on the whole real line, is called dilation analytic [11, 12] if $\widehat{V}$ is $H_{0}$-compact and the operator-valued function

$$
C(\theta)=u(\theta) \widehat{V} u(\theta)^{-1}\left(H_{0}+1\right)^{-1}
$$

extends to an analytic operator-valued function on the strip $\mathscr{S}(a)=\{z:-a<$ $\operatorname{Im}(z)<a\}$ for some $a>0$.

With $V$ as in (1.4), we define

$$
\widehat{V}(x)=V(|x|+1), \quad x \in \mathbf{R} .
$$

In the sequel we assume that $f$ is $C^{3}[1, \infty)$ so that $V$ is a differentiable function. Our main result is

Theorem 1.3. Suppose that $k$ and $V^{\prime}$ are short range and that $\widehat{V}$ is dilation analytic. Then all conclusions of Theorem 1.1(iv) remain valid.

Since, for $f(x)=\exp \left(-x^{\alpha}\right)$,

$$
\widehat{V}(x)=\frac{\alpha^{2}}{4}(|x|+1)^{2(\alpha-1)}-\frac{\alpha(\alpha-1)}{2}(|x|+1)^{\alpha-2}
$$

is certainly dilation analytic [11] if $0<\alpha<1$, the above theorem applies to the range of $\alpha$ not covered by Theorem 1.1 .

We finally remark that all the above-mentioned results have natural extensions to higher-dimensional nonsymmetric regions, as well as to manifolds with metric cusps at infinity $[2,6,8]$.

\section{Proof of TheOREM 1.3}

Let $H_{V}$ be the operator (1.4) with the Neumann boundary condition at 1 and denote by

$$
D=\left\{\psi: \psi \in C^{2}[1, \infty), \psi^{\prime}(1)=0\right\}
$$

its domain of essential selfadjointness. Let $J: L^{2}(R) \rightarrow L^{2}(\Omega)$ be the embedding $(J \phi)(x)=\phi(x) / \sqrt{2 f(x)}$, and denote $Q=1-J J^{*}$. 
The first ingredient in our argument is the following result of Davies and Simon [2].

Theorem 2.1. (i) If $\phi \in D$,

$$
\left\|(H+1)^{-1 / 2}\left(H J-J H_{V}\right) \phi\right\| \leq 2 \cdot\left\|f^{\prime} \phi\right\|_{2}+\left\|\left(\left(f^{\prime}\right)^{2} / f\right) \phi\right\|_{2}+C|\phi(1)|,
$$

where $C$ is a positive constant.

(ii) $Q(H+1)^{-1 / 2}$ is a compact operator in $L^{2}(\Omega)$.

(iii) If $g$ is a continuous function on $R \cup\{\infty\}, g(H) J-J g\left(H_{V}\right)$ is a compact operator in $L^{2}(\Omega)$.

We now use the decay of $V^{\prime}$ and the dilation analyticity of $\widehat{V}$ to obtain a useful decomposition of $V$ into a short-range part and a smooth long-range dilation analytic part [10]. $\widehat{V}$ can be decomposed as

$$
\widehat{V}(x)=\widehat{V}_{L}(x)+\widehat{V}_{S}(x),
$$

where $\widehat{V}_{L}(x)$ is a $C^{\infty}$ dilation analytic potential with all derivatives bounded,

$$
\widehat{V}^{\prime}(x)=O\left(|x|^{-1-\varepsilon}\right),
$$

and $\widehat{V}_{S}(x)$ is a short-range function. Both parts $\widehat{V}_{L}, \widehat{V}_{S}$ can be chosen to be even functions around 0 , and thus they induce the decomposition

$$
V(x)=V_{L}(x)+V_{S}(x),
$$

with obvious inheritance of properties. We refer to [10] for a proof of (2.1). $\widehat{V}_{L}$ is defined as the Weierstrass transform of $\widehat{V}$,

$$
\widehat{V}_{L}(x)=\frac{1}{2 \sqrt{\pi}} \int_{-\infty}^{\infty} \exp \left(-\frac{(x-y)^{2}}{4}\right) \widehat{V}(y) d y .
$$

Let

$$
\widehat{H}_{L}=H_{0}+\widehat{V}_{L}, \quad H_{L}=-\frac{d^{2}}{d x^{2}}+V_{L},
$$

where the second operator acts on $L^{2}([1, \infty), d x)$ with the Neumann boundary condition at 1 . The third ingredient in the argument consists of Perry's propagation estimates $[10,11]$ on $\exp \left(-i t \widehat{H}_{L}\right)$ (see also [1]), which automatically yield the propagation estimates on $\exp \left(-i t H_{L}\right)$. Let $A$ be a scale transformation around $x=1[1,10]$,

$$
A=\frac{1}{2}((x-1) \cdot p+p \cdot(x-1)),
$$

where $p=-i D=-i \partial / \partial x$ in the $x$-space representation. $A$ is essentially selfadjoint on $C_{0}^{\infty}(R), \sigma(A)=\sigma_{\mathrm{ac}}(A)=(-\infty, \infty)$, and $A$ leaves invariant the subspace of functions which are even around 1. By $P_{ \pm}$we denote the spectral projections of $A$ on $\pm(0, \infty)$ and by $P_{ \pm}^{a}$ the spectral projections on $\pm(a, \infty)$. Obviously, $P_{+}+P_{-}=1$. It is a standard result [12] that operators $\widehat{H}_{L}, H_{L}$ have no (strictly) positive eigenvalues, that $\sigma_{\text {sing }}\left(\widehat{H}_{L}\right)=\sigma_{\text {sing }}\left(H_{L}\right)=\varnothing$, and that $\sigma_{\mathrm{ac}}\left(\widehat{H}_{L}\right)=\sigma_{\mathrm{ac}}\left(H_{L}\right)=[0, \infty)$. Since $\widehat{V}$ is even around $0, \widehat{H}_{L}$ preserves the subspace of functions which are in its domain and are even around 0 . After translation by 1 , the restriction of $\widehat{H}_{L}$ to that subspace coincides with $H_{L}$. In 
the sequel we will use Enss' notation where $F(M)$ stands for the characteristic function of the set $M . P_{\mathrm{ac}}$ will denote the spectral projection on the absolutely continuous subspace of $H_{L}$. Viewing $H_{L}$ as a restriction of $\widehat{H}_{L}$ (keeping the above translation in mind) we have

Theorem 2.2. (i) Let $g$ be a $C^{\infty}$ function with support in $[\alpha, \beta], \alpha>0$. Then for any $\delta>0, N>0$, and $a \in \mathbf{R}$,

$$
\left\|F\left(1 \leq x<|t|^{1-\delta}\right) \exp \left(i t H_{L}\right) g\left(H_{L}\right) P_{ \pm}^{a}\right\|=O\left(|t|^{-N}\right)
$$

as $t \rightarrow \pm \infty$.

(ii) $\mathrm{s}-\lim _{t \rightarrow \pm \infty} P_{ \pm}^{a} \exp \left(-i t H_{L}\right)=0$.

The above theorem is a result by Perry $[10,11]$.

Since $H_{L}$ plays the role of the "free Laplacian" in the discussion below, it is worthwhile to rephrase part (i) of Theorem 2.1 as

$$
\left\|(H+1)^{-1 / 2}\left(J H-J H_{L}\right) \phi\right\| \leq 2 \cdot\left\|f^{\prime} \phi\right\|_{2}+\left\|\left(\left(f^{\prime}\right)^{2} / f\right) \phi\right\|_{2}+\left\|V_{L} \phi\right\|_{2}+C|\phi(1)|
$$

and to note, concerning part (iii), that $g(H) J-J g\left(H_{L}\right)$ is a compact operator in $L^{2}(\Omega)$ (since $g\left(H_{V}\right)-g\left(H_{L}\right)$ is compact).

Since $V_{L}$ is bounded from below, without loss of generality we can assume $V_{L} \geq 0$. The final technical lemma is the following

\section{Lemma 2.3.}

$$
\left\|(H+1)^{-1 / 2}\left(H J-J H_{L}\right)\left(H_{L}+1\right)^{-1} F(x>R)\right\|=O\left(R^{-1-\varepsilon}\right) .
$$

The proof is standard [1, Lemma 5.4], using Theorem 2.1(i) and the fact that $k, V_{S}$ are short-range.

From now on, the proof follows line-by-line the argument of Davies and Simon [2]. We give the details in the appendix for the reader's convenience.

\section{APPENDIX}

Step 1. s-lim $\lim _{t \rightarrow \infty}(H+1)^{-1 / 2} \exp (i t H) J \exp \left(-i t H_{L}\right) P_{\mathrm{ac}}$ exists. Let us consider only the limit $t \rightarrow \infty$; a similar consideration applies to the other one. By Cook's criterion, it suffices to show that

$$
\int_{0}^{\infty}\left\|(H+1)^{-1 / 2}\left(H J-J H_{L}\right) \exp \left(-i t H_{L}\right) g\left(H_{L}\right) P_{+}^{a}\right\| d t<\infty,
$$

where $g$ is as in Theorem 2.2, since $\bigcup_{a, g} \operatorname{Ran} g\left(H_{L}\right) P_{+}^{a}$ is dense in $\mathscr{H}_{\mathrm{ac}}\left(H_{L}\right)$. Denote

$$
A(t)=\left\|(H+1)^{-1 / 2}\left(H J-J H_{L}\right) \exp \left(-i t H_{L}\right) g\left(H_{L}\right) P_{+}^{a}\right\| .
$$

Choosing $\delta<\varepsilon$ in (2.2), we estimate, using Theorem 2.2 and Lemma 2.3,

$$
\begin{aligned}
A(t) \leq & \left\|(H+1)^{-1 / 2}\left(H J-J H_{L}\right)\left(H_{L}+1\right)^{-1} F\left(x \geq t^{-1-\delta}\right)\right\| \\
& +\left\|F\left(1 \leq x<t^{-1-\delta}\right) \exp \left(-i t H_{L}\right)\left(H_{L}+1\right) g\left(H_{L}\right) P_{+}^{a}\right\| \\
\leq & O\left(t^{-1-\varepsilon+\delta}\right) .
\end{aligned}
$$

Step 2. The wave operators $\Omega^{ \pm}=\mathrm{s}-\lim _{t \rightarrow \mp \infty} \exp (i t H) J \exp \left(-i t H_{L}\right) P_{\mathrm{ac}}$ exist. $(H+1)^{-1 / 2} J-J\left(H_{L}+1\right)^{-1 / 2}$ is compact by Theorem 2.1 , since

$$
\begin{aligned}
& (H+1)^{-1 / 2} J-J\left(H_{L}+1\right)^{-1 / 2} \\
& \quad=(H+1)^{-1 / 2} J-J\left(H_{V}+1\right)^{-1 / 2}+J\left(H_{V}+1\right)^{-1 / 2}-J\left(H_{L}+1\right)^{-1 / 2} .
\end{aligned}
$$


Thus

$$
\text { s- } \lim _{t \rightarrow \mp \infty} \exp (-i t H)\left((H+1)^{-1 / 2} J-J\left(H_{L}+1\right)^{-1 / 2}\right) \exp \left(-i t H_{L}\right) P_{\mathrm{ac}}=0 .
$$

By Step $1, \Omega^{ \pm} \phi$ exist if $\phi \in \operatorname{Ran}\left(H_{L}+1\right)^{-1 / 2} P_{\mathrm{ac}}$, and the last set coincides with $\mathscr{X}_{\mathrm{ac}}\left(H_{L}\right)$.

Step 3. $(H+1)^{-1 / 2}\left(\Omega^{ \pm}-J\right) g\left(H_{L}\right) P_{ \pm}$is a compact operator.

First, since $\left(H_{L}+1\right)^{-1 / 2}\left(\exp \left(i t H_{L}\right) J \exp \left(-i t H_{L}\right)-J\right) g\left(H_{L}\right) P_{ \pm}$converges in the operator norm as $t \rightarrow \pm \infty$, it suffices to show that each of these operators is compact. On the other hand, they are integrals of operators of the form

$$
(H+1)^{-1 / 2}\left(H J-J H_{L}\right) \exp \left(-i t H_{L}\right) g\left(H_{L}\right) P_{ \pm}
$$

and thus it suffices to show that the operators (A.1) are compact. But that is a consequence of part (iii) of Theorem 2.1 and of the fact that $V_{S}$ is short-range.

Step 4. If $\phi_{n} \in \mathscr{H}_{a c}(H)^{\perp}$, with $\left\|(H+1) \phi_{n}\right\|$ bounded and $(H+1)^{1 / 2} \phi_{n} \rightarrow 0$ weakly, then $\left\|\phi_{n}\right\| \rightarrow 0$ as $n \rightarrow \infty$.

First, $\left(\Omega^{ \pm}\right)^{*} \phi_{n}=0$ for all $n$, and thus for any $C_{0}^{\infty}((0, \infty))$ function $g$, Step 3 yields

$$
P_{ \pm} g\left(H_{L}\right) J^{*} \phi_{n} \rightarrow 0 \text {. }
$$

Since $P_{+}+P_{-}=1$ and $g\left(H_{L}\right) J^{*}-J^{*} g(H)$ is a compact operator, we obtain that $J^{*} g(H) \phi_{n} \rightarrow 0$. Since $\left\|(H+1) \phi_{n}\right\|$ is bounded, we estimate

$$
\left\|g(H) \phi_{n}-\phi_{n}\right\| \leq C \cdot\left\|(g(H)-1) \cdot(H+1)^{-1}\right\| .
$$

Since $g$ is arbitrary, the left-hand side of (A.2) can be made arbitrarily small, and thus $J^{*} \phi_{n} \rightarrow 0$, and so $J J^{*} \phi_{n} \rightarrow 0$. By Theorem 2.1, $Q \phi_{n}=$ $\left(1-J J^{*}\right) \phi_{n} \rightarrow 0$, and we conclude that $\phi_{n} \rightarrow 0$.

Step 5. $\sigma_{\text {sing }}(H)=\varnothing$ and in any finite interval $H$ has only finitely many eigenvalues. If any of the statements is not valid, we can construct an orthonormal sequence $\phi_{n}$ so that $\phi_{n} \in \mathscr{H}_{\mathrm{ac}}(H)^{\perp},\left\|(H+1) \phi_{n}\right\|$ is bounded, and $(H+1)^{1 / 2} \phi_{n} \rightarrow 0$ weakly. Step 4 implies then that $\phi_{n} \rightarrow 0$, which contradicts the fact that the sequence $\phi_{n}$ is orthonormal.

Step 6. $\sigma_{\mathrm{ac}}(H)$ has multiplicity one. It suffices to show that $\operatorname{Ran} \Omega^{+}=$ $\mathscr{H}_{\text {ac }}(H)$. Suppose that it is not, namely, that we can find a nonzero vector $\phi \in$ $\mathscr{H}_{\text {ac }}(H) \cap\left(\operatorname{Ran} \Omega^{+}\right)^{\perp} \cap D(H)$. Define $\phi_{n}=\exp (-i n H) \phi$. Part (ii) of Theorem 2.2 yields

$$
P_{ \pm}\left(\Omega^{ \pm}\right)^{*} \phi_{n}=P_{ \pm} \exp \left(-i n H_{L}\right) P_{\mathrm{ac}}\left(\Omega^{ \pm}\right)^{*} \phi \rightarrow 0 \quad \text { as } n \rightarrow \infty .
$$

Thus, as in Step 4, $\left\|\phi_{n}\right\| \rightarrow 0$, and we derive that $\phi=0$, a contradiction.

Step 7. There exists a discrete set of embedded eigenvalues. This is a consequence of symmetry of $\Omega$ with respect to the axes $y=0$. Let $E$ be the subspace of $L^{2}(\Omega)$ consisting of those functions which are even under reflection $(x, y) \rightarrow(x,-y)$. That subspace is invariant under $Q$ and $H$, and thus $H$ restricted to it has a compact resolvent by Theorem 2.1 .

\section{ACKNOWLEDGMENTS}

It is a pleasure to thank F. Geszetsy, L. Romans, and B. Simon for valuable discussions. 


\section{REFERENCES}

1. H. Cycon, R. Froese, W. Kirsch, and B. Simon, Schrödinger operators, Springer-Verlag, Heidelberg, 1987.

2. E. B. Davies and B. Simon, Spectral properties of the Neumann Laplacian of horns, Geometry and Functional Analysis (to appear).

3 V. Enss, Asymptotic completeness for quantum mechanical potential scattering, I. Short range potentials, Comm. Math. Phys. 61 (1978), 285-291.

4. __ Asymptotic completeness for quantum mechanical potential scattering, II. Singular and long range potentials, Ann. Phys. 119 (1979), 117-132.

5. W. Evans and D. Harris, Sobolev embeddings for generalized ridge domains, Proc. London Math. Soc. (3) 54 (1987), 141-175.

6. R. Froese and P. Hislop, Spectral analysis of second-order elliptic operators on noncompact manifolds, Duke Math. J. 58 (1989), 103-129.

7. R. Hempel, L. A. Seco, and B. Simon, The essential spectrum of the Neumann Laplacian on some bounded singular domains, J. Funct. Anal. 102 (1991), 448-483.

8. V. Jakšić, S. Molčanov, and B. Simon, Eigenvalue asymptotics of the Neumann Laplacian of regions and manifolds with cusps, J. Funct. Anal. 106 (1992), 59-79.

9. E. Mourre, Link between the geometrical and the spectral transformation approaches in scattering theory, Comm. Math. Phys. 68 (1979), 91-94.

10. P. Perry, Propagation of states in dilation analytic potentials and asymptotic completness, Comm. Math. Phys. 81 (1981), 243-259.

11. __ Scattering theory by the Enss Method, Harwood, New York, 1983.

12. M. Reed and B. Simon, Methods of modern mathematical physics, IV. Analysis of operators, Academic Press, New York, 1978.

13. F. Rellich, Das Eigenwert problem von $\Delta \lambda+\lambda \mu=0$, Studies and Essays, Interscience, New York, 1948, pp. 329-344.

14. B. Simon, The Neumann Laplacian of a jelly roll, Proc. Amer. Math. Soc. 114 (1992), 783-785.

Division of Physics, Mathematics and Astronomy, California Institute of Technology, Pasadena, California 91125 $1 \mathrm{~A} 1$

Current address: Department of Mathematics, University of Toronto, Toronto, Canada M5S

E-mail address: VOJKAN@MATH.TORONTO.EDU 